\title{
ON THE DYNAMICS OF A CHARGED PARTICLE IN A SPIRAL FIELD
}

Received:

\author{
Joseph A. Burns* and Gerald Halpern* \\ Laboratory for Theoretical Studies, NASA - Goddard Space Flight Center \\ Greenbelt, Maryland 20771
}

Mĩay $19 \overline{1} 68$

*NAS-NRC Resident Research Associates

Running Title: Charged Particles in a Spiral Field

GODDARD SPACE FLIGHT CENTER

Greenbelt, Maryland 


\title{
PRECEDING PAGE BLANK NOT FILMED.
}

\author{
ON THE DYNAMICS OF A CHARGED PARTICLE \\ IN A SPIRAL FIELD \\ Joseph A. Burns and Gerald Halpern \\ Laboratory for Theoretical Studies, NASA \\ Goddard Space Flight Center
}

\begin{abstract}
The adiabatic guiding center motion of an energetic charged particle in an idealized representation of the interplanetary magnetic field is discussed. Expressions are presented for all drift velocity components. A comparison of these drifts establishes that, for the cases of physical interest, the electric drift velocity is by far the dominant drift velocity.

Considering only the electric drift velocity, an expression is derived for the time of flight of a particle leaving the sun. Similarly, it is shown analytically that the particles remain attached to the same field line on which they left the sun; thus indicating their point of origin. High energy unscattered particles are shown to be rapidly channelled down the field lines, resulting in a highly anisotropic particle distribution in the vicinity of the earth. These analytic results are in excellent agreement with numerical integration of the complete drift equation and compare favorably with the experimental data.
\end{abstract}




\section{ON THE DYNAMICS OF A CHARGED PARTICLE \\ IN A SPIRAL FIELD}

\section{INTRODUCTION}

Analytic solutions are presented which describe the adiabatic guiding center motion of an energetic charged particle in the idealized spiral model of the interplanetary magnetic field (Parker, 1958). These solutions have simple physical interpretation and show the dependence of the motion on the various solar wind and particle parameters. Illustrative figures drawn for typical values of these parameters are also displayed.

To estimate the validity of the approximate method used herein, we solved numerically the complete guiding center equation of motion. Our analytic solutions were confirmed in all cases by these computer results and agreed with most of the numerical findings of Winge and Coleman (1968). In addition, the analytic approach provides a generalization of the computer results.

The solutions apply to all charged particles that are not appreciably scattered by magnetic irregularities in the interplanetary field. While many experimental results can be understood from the analysis herein, this paper is not meant to describe the exact motion of a charged particle from the sun to earth; disagreements between our results and experiments may arise because of the more complex and irregular structure of the actual magnetic field.

\section{MODEL OF THE SPIRAL FIELD}

We use the same model (Parker, 1958) for the interplanetary magnetic field as was discussed in some detail by Winge and Coleman (1968). The sun's surface 
magnetic field is combed out into interplanetary space by the radial expansion of the perfectly conducting solar wind. Since the magnetic flux lines lie along solar wind streamlines when viewed in a coordinate system rotating with the sun, the shape of the field is determined beyond $r_{0}$, the radius at which corotation ceases.

The magnetic field is shown in Figure 1 and is written in an inertial, heliocentric coordinate system as

$$
B=B_{o}\left(r_{o} / r\right)^{2}\left[e_{r}-\Omega r \sin \theta / N_{p} e_{\phi}\right]
$$

where the e's are the unit vectors in the $(r, \theta, \phi)$ spherical polar coordinate directions with $\theta$ the colatitude measured from the sun's rotation axis and $\phi$ the longitude, which is positive in the direction of the sun's rotation. $B_{o}$ is the radial component of the field at $r=r_{o}, \Omega$ is the angular velocity of the sun and $v_{p}$ is the velocity of the solar wind, which is assumed constant and radial. For the remainder of this paper in numerical examples, we shall choose $r_{0}=1.38 \times 10^{11} \mathrm{~cm}$ or twice the radius of the sun, $B_{0}=5 \times 10^{-5}\left(1 \mathrm{AU} / \mathrm{r}_{0}\right)^{2}$ gauss $=0.591$ gauss and $\Omega=2.90 \times 10^{-6} \mathrm{rad}-\mathrm{sec}^{-1} \cdot \mathrm{V}_{\mathrm{p}}$ shall be a constant parameter in the problem.

The magnetic field may be simply expressed by using a unit vector $e_{1}$, which lies along the magnetic field, as

$$
B=B e_{1}=B_{0}\left(r_{0} / r\right)^{2} \sec a e_{1}
$$

where

$$
\mathbf{e}_{1}=\cos \alpha \mathbf{e}_{\mathrm{r}}-\sin \alpha \mathbf{e}_{\phi}
$$

The angle $a$ between $B$ and $e_{r}$ is given by 


$$
\alpha=\tan ^{-1}\left(-B_{\phi} / B_{r}\right)=\tan ^{-1}(r / a)
$$

with the quantity a being a convenient scaling length:

$$
a=v_{p} /(\Omega \sin \theta)
$$

It is the radius at which the magnetic field makes an angle of $45^{\circ}$ with respect to

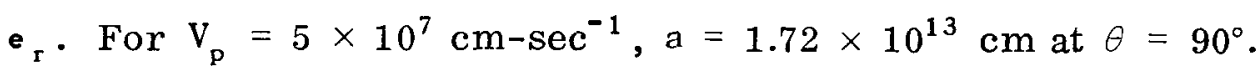

In the inertial system there is an electric field,

$$
\begin{aligned}
E & =-\frac{1}{c} v_{p} \times B=-(1 / c) \Omega \sin \theta B_{o} r_{o}^{2} / \mathbf{r} e_{\theta} \\
& =-\frac{1}{c} v_{p} r B \cos a / a e_{\theta}
\end{aligned}
$$

where $\mathrm{c}$ is the velocity of light. This electric field results from the convection of the magnetic field lines out with the solar wind speed. It should be noted that no electric field would be seen in a reference system moving with the solar wind plasma or in a reference system rotating with the sun.

\section{CHARGED PARTICLE DYNAMICS}

To simplify the discussion of the motion of a charged particle in a nonuniform electromagnetic field we use guiding center theory, that is, we concern ourselves with the motion of the guiding center, the point about which the particle may be considered to be gyrating. The particle itself may be shown to be nearby. The conditions under which guiding center theory is valid have been discussed by $\underline{\text { Alfvén and Fälthammar (1963) and presented for our problem by Winge and }}$ Coleman (1968) as the criteria for adiabatic invariance. These criteria were shown to be satisfied for all but extremely high energy particles. 
The guiding center velocity $V$ may be written in terms of a velocity $V_{11}$ along the field and a velocity $v_{d}$, the drift velocity, perpendicular to the field, as

$$
\mathbf{v}=\mathbf{v}_{11}+\mathbf{v}_{\mathbf{d}}
$$

The actual particle velocity is $V$ plus $v_{c}$, the velocity due to the gyration motion about the guiding center.

Northrop (1963) has expressed the drift velocity of a particle with mass $\mathrm{m}$, charge e and magnetic moment $\mu$ as

$$
V_{d}=\frac{e_{1}}{B} \times\left[-c E+\frac{\mu c}{e} \nabla B+\frac{m c}{e}\left(v_{11} \frac{d e_{1}}{d t}+\frac{d U_{E}}{d t}-g\right)\right]
$$

where the electric drift velocity

$$
\mathbf{U}_{\mathbf{E}}=\mathrm{CE} \times \mathbf{e}_{\mathbf{1}} / \mathrm{B}
$$

and the gravitational acceleration

$$
g=-M_{\odot} G_{r} / r^{2}
$$

$M_{\odot}$ and $G$ being the solar mass and the universal gravitational constant, respectively. The first two terms of the right hand member of Equation (8) are readily identified as the electric drift and the gradient B drift, while the remaining three terms account for the acceleration drift.

Some care must be taken in the evaluation of the magnetic moment $\mu$, which is an adiabatic invariant of the motion. The Appendix discusses this point and shows why for most cases we choose 


$$
\mu=\frac{\mathrm{m} \mathrm{V}_{\mathrm{T}}^{2} \sin ^{2}}{2 \mathrm{~B}} \psi=\frac{\mathrm{m} \mathrm{V}_{\mathrm{c}}^{2}}{2 \mathrm{~B}}
$$

where $\mathrm{V}_{\mathrm{T}}$ is the total particle velocity and $\psi$ is the pitch angle, that is, the angle between the particle's total velocity vector and the magnetic field.

\section{DRIFT VELOCITIES IN A SPIRAL FIELD}

\section{A. Expressions for Velocities}

Using the representation of the spiral field given by Equations (1) and (6), we will now obtain an explicit formula for each drift term in Equation (8). This will permit us to demonstrate that the electric drift is by far the largest term, which will enable us to give a complete and accurate, although approximate, description of the guiding center motion.

The electric drift is

$$
\mathbf{U}_{\mathrm{E}}=\mathrm{c} \mathbf{E} \times \mathbf{e}_{1} / \mathrm{B}=\frac{\mathrm{V}_{\mathrm{p}} \mathrm{r}}{\mathrm{r}^{2}+\mathrm{a}^{2}}\left(\mathrm{r} \mathbf{e}_{\mathbf{r}}+\mathrm{a} \mathbf{e}_{\phi}\right)
$$

The gradient of the field magnitude is

$$
\nabla B=-\frac{B}{r\left(r^{2}+a^{2}\right)}\left[\left(r^{2}+2 a^{2}\right) e_{r}-r^{2} \cot \theta e_{\theta}\right]
$$

which gives the gradient $B$ drift as

$$
\mathbf{U}_{\mathrm{B}}=\frac{\mathbf{e}_{1}}{\mathrm{~B}} \times\left(\frac{\mu \mathrm{c}}{\mathrm{e}}\right) \nabla \mathrm{B}=\frac{\mu \mathrm{c}}{\mathrm{e}}\left(\mathrm{r}^{2}+\mathrm{a}^{2}\right)^{-3 / 2}\left[\mathrm{r}^{2} \cot \theta \mathbf{e}_{\mathrm{r}}+\left(\mathrm{r}^{2}+2 \mathrm{a}^{2}\right) \mathbf{e}_{\theta}+\mathrm{ra} \cot \theta \mathbf{e}_{\phi}\right]
$$

For a steady state problem, the operator $\frac{d}{d t}$ in Equation (8) reduces to lowest order to the convective derivative along $\mathbf{B}$ :

$$
\mathrm{V}_{11}\left(\mathbf{e}_{1} \cdot \boldsymbol{\nabla}\right)=\mathrm{V}_{11} \frac{\partial}{\partial \mathrm{s}}
$$


Hence the acceleration terms are

$$
\begin{gathered}
\underline{U}_{A 1}=\frac{m c}{e} \frac{e_{1}}{B} \times V_{11} \frac{d e_{1}}{d t}=\frac{m c}{e B} V_{11}^{2}\left(r^{2}+a^{2}\right)^{-3 / 2}\left(-r^{2} \cot \theta e_{r}\right. \\
\left.\quad+\left(r^{2}+2 a^{2}\right) e_{\theta}-r a \cot \theta e_{\phi}\right) \\
\underline{U}_{A 2}=\frac{m c}{e} \frac{e_{1}}{B} \times \frac{d U_{E}}{d t}=\frac{m c}{e B} \frac{V_{p} V_{11} a}{\left(r^{2}+a^{2}\right)^{2}}\left(r^{2} \cot \theta e_{r}-a^{2} e_{\theta}+r a \cot \theta e_{\phi}\right)(14) \\
\underline{U}_{G}=-\frac{m c}{e} \frac{e_{1}}{B} \times g=-\frac{m c M_{\odot} G}{e B r\left(r^{2}+a^{2}\right)^{1 / 2}} e_{\theta}
\end{gathered}
$$

We can combine results (10) through (15) by defining the total drift velocity as

$$
\mathbf{v}_{\mathrm{d}}=\mathrm{V}_{\mathrm{dr}} \mathbf{e}_{\mathrm{r}}+\mathrm{V}_{\mathrm{d} \theta} \mathbf{e}_{\theta}+\mathrm{V}_{\mathrm{d} \phi} \mathbf{e}_{\phi}
$$

where

$$
\begin{gathered}
V_{d r}=\frac{V_{p} r^{2}}{r^{2}+a^{2}}+\frac{\mu c}{e} r^{2}\left(r^{2}+a^{2}\right)^{-3 / 2} \cot \theta-\frac{m c V_{I I}^{2}}{e B} r^{2}\left(r^{2}+a^{2}\right)^{-3 / 2} \cot \theta \\
\quad+\frac{m c V_{p} V_{11} a r^{2}}{e B}\left(r^{2}+a^{2}\right)^{-2} \cot \theta \\
V_{d \phi}=V_{d r} a / r \\
V_{d \theta}=\frac{\mu c}{e}\left(r^{2}+2 a^{2}\right)\left(r^{2}+a^{2}\right)^{-3 / 2}+\frac{m c V_{11}^{2}}{e B}\left(r^{2}+2 a^{2}\right)\left(r^{2}+a^{2}\right)^{-3 / 2} \\
-\frac{m c V_{p} V_{11} a^{3}}{e B}\left(r^{2}+a^{2}\right)^{-2}-\frac{m c M_{0} G}{e B r}\left(r^{2}+a^{2}\right)^{-1 / 2}
\end{gathered}
$$


It is of interest to note that $\mathrm{V}_{\mathrm{d} r}$ and $\mathrm{V}_{\mathrm{d} \phi}$ for particles in the equatorial plane are due entirely to the electric drift and that all other drifts are proportional to the mass of the particle.

\section{B. Comparison of Velocities}

To demonstrate that $U_{E}$ is the dominant drift, we proceed in the following manner: 1.) we find the largest contributor to the $\theta$ drift; 2.) we show that the $(r, \phi)$ drifts are at most comparable to their $\theta$ counterparts; and 3.) we compare the term in part 1.) to $U_{E}$ and show it is small.

1.) We first consider the drift velocity in the $\theta$ direction, given by Equation (19).

We compare the various terms to the second term $\mathrm{U}_{\mathrm{A} 1 \theta}$ :

$$
\frac{\mathrm{U}_{\mathrm{B} \theta}}{\mathrm{U}_{\mathrm{A} 1 \theta}}=\left(\frac{\mathrm{V}_{\mathrm{c}}}{\mathrm{V}_{11}}\right)^{2}, \frac{\mathrm{U}_{\mathrm{A} 2 \theta}}{\mathrm{U}_{\mathrm{A} 1 \theta}}=-\mathrm{V}_{\mathrm{p}} \mathrm{a}^{3} /\left[\mathrm{V}_{11}\left(\mathrm{r}^{2}+2 \mathrm{a}^{2}\right)\left(\mathrm{r}^{2}+\mathrm{a}^{2}\right)^{1 / 2}\right]
$$

and

$$
\frac{U_{G \theta}}{U_{A 1 \theta}}=-\frac{M_{\odot} G}{r V_{11}^{2}} \frac{\left(r^{2}+a^{2}\right)}{\left(r^{2}+2 a^{2}\right)}
$$

It may easily be shown from these expressions that $\mathrm{U}_{\mathrm{A} 1 \theta}$ is the dominant $\theta$ drift except when the initial pitch angle is near $90^{\circ}$.

2.) We will now compare the various terms of the total $(r, \phi)$ drift

$$
\mathrm{V}_{\mathrm{r} \phi} \equiv\left[\mathrm{V}_{\mathrm{r}}^{2}+\mathrm{v}_{\phi}\right]^{1 / 2}
$$

with their corresponding $\theta$ drifts: 


$$
\begin{aligned}
& \mathrm{U}_{\mathrm{B}}: \mathrm{V}_{\mathrm{r} \phi} / \mathrm{V}_{\theta}=\mathrm{r} \cot \theta\left(\mathrm{r}^{2}+\mathrm{a}^{2}\right)^{1 / 2} /\left(\mathrm{r}^{2}+2 \mathrm{a}^{2}\right) \\
& \mathrm{U}_{\mathrm{A} 1}: \mathrm{V}_{\mathrm{r} \phi} / \mathrm{V}_{\theta}=-\mathrm{r} \cot \theta\left(\mathrm{r}^{2}+\mathrm{a}^{2}\right)^{1 / 2} /\left(\mathrm{r}^{2}+2 \mathrm{a}^{2}\right) \\
& \mathrm{U}_{\mathrm{A} 2}: \mathrm{V}_{\mathrm{r} \phi} / \mathrm{V}_{\theta}=-\mathrm{r} \cot \theta\left(\mathrm{r}^{2}+\mathrm{a}^{2}\right)^{1 / 2} / \mathrm{a}^{2} \\
& \mathrm{U}_{\mathrm{G}}: \mathrm{V}_{\mathrm{r} \phi} / \mathrm{V}_{\theta}=0
\end{aligned}
$$

These ratios are all of order 1 or less since $r$ is of the same order as a

3.) Comparing the total electric drift velocity $U_{E}$ to $U_{A 1 \theta}$, we find

$$
\frac{\mathrm{U}_{\mathrm{A} 1 \theta}}{\mathrm{U}_{\mathrm{E}}}=\frac{\mathrm{mcV_{11 } ^ { 2 }}}{\mathrm{eBV_{ \textrm {p } } \mathrm { r }}}\left(\frac{\mathrm{r}^{2}+2 \mathrm{a}^{2}}{\mathrm{r}^{2}+\mathrm{a}^{2}}\right)
$$

To a good approximation $V_{11}$ is constant. Thus for $V_{p}=5 \times 10^{7} \mathrm{~cm}^{-\mathrm{sec}^{-1}}$ and protons with $\mathrm{V}_{\mathrm{I}}=3 \times 10^{9} \mathrm{~cm}-\mathrm{sec}^{-1}$

$$
\frac{\mathrm{U}_{\mathrm{A} 1 \theta}}{\mathrm{U}_{\mathrm{E}}}=5 \times 10^{-20} \frac{\mathrm{ar}\left(2 \mathrm{a}^{2}+\mathrm{r}^{2}\right)}{\left(\mathrm{a}^{2}+\mathrm{r}^{2}\right)^{3 / 2}}
$$

This is a maximum at $r=2^{1 / 2}$ a and there is of order $10^{-6}$. For cases in which the initial pitch angle is close to $90^{\circ}, \mathrm{U}_{\mathrm{B} \theta}$ will be the largest contributor to 1.$)$ when the particle is close to the sun. In this case $V_{c}^{2}$ would replace $V_{11}^{2}$ in Equation (20) and the argument would proceed as before.

Thus the electric drift dominates this problem and we are able to write that, to a very good approximation, 


$$
v_{d} \approx U_{E}=\frac{r V_{p}}{r^{2}+a^{2}}\left(r e_{r}+a e_{\phi}\right)
$$

We note there is no $\theta$ component.

\section{PARTICLE MOTION}

In this section we will derive expressions which describe the motion of a charged particle's guiding center in the spiral magnetic field. Specifically, we will consider an energetic particle (e.g., proton) of energy $\varepsilon$ and initial pitch angle $\psi_{0}$ which is injected from the sun into interplanetary space at $r=r_{0}$, colatitude $\theta_{0}$ and longitude $\phi_{0}$. Our task is to obtain $r(t)$ and $\phi(t)$ as functions of these initial parameters and the solar wind velocity. Since $\mathrm{v}_{\theta} \approx 0, \theta(\mathrm{t})=\theta_{0}$, within our approximation.

\section{A. Radial Motion}

The radial position of the guiding center is the solution of the differential equation

$$
\frac{\mathrm{dr}}{\mathrm{dt}} \equiv \mathrm{V}_{\mathrm{r}}=\mathrm{V}_{\mathrm{dr}}+\mathrm{V}_{\mathrm{H}_{\mathrm{r}}}
$$

where

$$
V_{d r}=V_{p} r^{2} /\left(r^{2}+a^{2}\right)
$$

and

$$
V_{H_{r}}=V_{11} \cos \alpha=V_{11} a /\left(r^{2}+a^{2}\right)^{1 / 2}
$$

We use conservation of energy and the adiabatic invariance of the magnetic moment to determine $V_{11}(r)$. The particle's kinetic energy is a conserved quantity because changes in the gravitational energy are entirely negligible and 
because the electric potential energy is automatically a constant of the motion since the particle moves on a surface of constant $\theta$. If $\mathrm{V}_{\mathrm{T}} \equiv(2 \mathcal{E} / \mathrm{m})^{1 / 2}$ is the initial speed of the particle, then

$$
\mathrm{V}_{\mathrm{T}}^{2}=\mathrm{V}_{11}^{2}+\mathrm{V}_{\mathrm{c}}^{2}+\mathrm{V}_{\mathrm{d}}^{2}
$$

$\mathrm{V}_{d}^{2}(r)$ is given by Equation (21) and $V_{c}^{2}(r)=\frac{2 \mu}{m} B(r)$ from Equation (9). Using Equations (2) and (9)

$$
V_{c}^{2}(r)=V_{T}^{2} \frac{r_{0}^{2}}{r^{2}}\left(\frac{r^{2}+a^{2}}{r_{0}^{2}+a^{2}}\right)^{1 / 2} \sin ^{2} \psi_{0}
$$

Thus Equation (25) reads

$$
V_{11}=\left\{V_{T}^{2}\left[1-\frac{r_{0}^{2}}{r^{2}}\left(\frac{r^{2}+a^{2}}{r_{0}^{2}+a^{2}}\right)^{1 / 2} \sin ^{2} \psi_{0}\right]-\frac{V_{p}^{2} r^{2}}{r^{2}+a^{2}}\right\}^{1 / 2}
$$

Using this expression for $V_{n}(r)$ in Equation (22) gives, in principle, $r(t)$. Unfortunately, we have not been able to perform the required integral, but some well founded approximations will enable us to arrive at an explicit representation of $t(r)$.

We first neglect $V_{d}^{2}$ compared to $V_{T}^{2}$ since $V_{d}^{2}<V_{p}^{2}<<V_{T}^{2}$ for all particles under consideration. For example, a $1 \mathrm{Mev}$ proton has $\mathrm{V}_{\mathrm{T}}=1.38 \times 10^{9} \mathrm{~cm}^{-\mathrm{sec}^{-1}}$ while a typical solar wind velocity is $5.00 \times 10^{7} \mathrm{~cm}_{-\mathrm{sec}^{-1}}$. Next, noting that $\mathrm{r}_{0}^{2}<<\mathrm{a}^{2}$ for all $\theta$, we can replace Equation (26) by the approximate form

$$
\mathrm{V}_{\mathrm{c}}^{2} \approx \mathrm{V}_{\mathrm{T}}^{2} \mathrm{r}_{0}^{2} \sin ^{2} \psi_{0} / \mathrm{r}^{2}
$$


Differences between this and the more exact Equation (26) are significant only for $\mathrm{r} \gtrsim \mathrm{a}$, when $\mathrm{V}_{\mathrm{c}}^{2}<<\mathrm{V}_{\mathrm{T}}^{2}$. Thus

$$
\mathrm{V}_{n} \approx \mathrm{V}_{\mathrm{T}}\left[1-\mathrm{r}_{0}^{2} \sin ^{2} \psi_{0} / \mathrm{r}^{2}\right]^{1 / 2}
$$

Using similar arguments, we neglect $V_{d r}$ compared to $V_{n_{r}}$ and obtain the radial equation of motion

$$
\frac{\mathrm{dr}}{\mathrm{d} t} \approx \mathrm{V}_{\mathrm{H}_{\mathrm{r}}}=\mathrm{V}_{\mathrm{T}} \frac{\mathrm{a}}{\mathrm{r}} \frac{\left(\mathrm{r}^{2}-\mathrm{r}_{0}^{2} \sin ^{2} \psi_{0}\right)^{1 / 2}}{\left(\mathrm{r}^{2}+\mathrm{a}^{2}\right)^{1 / 2}}
$$

which may be integrated to give

$$
\begin{aligned}
t(r)= & \frac{1}{2 a V_{T}}\left\{\left(r^{2}-r_{0}^{2} \sin ^{2} \psi_{0}\right)^{1 / 2}\left(r^{2}+a^{2}\right)^{1 / 2}-r_{0} \cos \psi_{0}\left(r_{0}^{2}+a^{2}\right)\right. \\
& \left.+\left(a^{2}+r_{0}^{2} \sin ^{2} \psi_{0}\right) \ln \left[\frac{\left(r^{2}+a^{2}\right)^{1 / 2}+\left(r^{2}-r_{0}^{2} \sin ^{2} \psi_{0}\right)^{1 / 2}}{\left(r_{0}^{2}+a^{2}\right)^{1 / 2}+r_{0} \cos \psi_{0}}\right]\right\}
\end{aligned}
$$

This gives the flight time for a particle to reach a distance $r$ from the sun if it is injected at $r_{0}$ with velocity $V_{T}$ and pitch angle $\psi_{0}$. It is plotted for various initial conditions in Figure 2.

If we consider $r_{0}<r$, a, then Equation (31) for $\theta=90^{\circ}$ simplifies to

$$
t(r) \approx\left(\frac{m}{2 \xi}\right)^{1 / 2} \frac{r}{2}\left\{\left(1+\frac{\Omega^{2} r^{2}}{v_{p}^{2}}\right)^{1 / 2}+\frac{v_{p}}{\Omega r} \ln \left[\frac{\Omega r}{v_{p}}+\left(1+\frac{\Omega^{2} r^{2}}{v_{p}^{2}}\right)^{1 / 2}\right]\right\}
$$


It is easily shown that for large $r, t \sim r^{3 / 2}$, which agrees with the asymptotic slope of $3 / 2$ seen in Figure 2 .

Lin and Anderson (1967) have tabulated over 25 prompt solar electron (>40 Kev) events for the years 1964-1966. The shortest travel time recorded for the electrons was 1500 seconds, while the longest was 5000 seconds. A travel time of 2000 seconds was typical. We find that $40 \mathrm{Kev}$ electrons would move from $r_{0}$ to the earth in our model in 1950 seconds, 1550 seconds and 1400 seconds for $V_{p}=200 \mathrm{~km}-\mathrm{sec}^{-1}, 350 \mathrm{~km}-\mathrm{sec}^{-1}$ and $500 \mathrm{~km}-\mathrm{sec}^{-1}$, respectively. These results are nearly independent of the initial pitch angle and, since they deal with the lowest energy particle measured, they are the longest travel times that would be measured according to our theory. The fact that larger travel times are measured indicates that path length is longer than assumed (a more irregular field) and/or injection of the particles at the connecting field line takes place later in the event. The latter explanation would mean that the particles either take some time to diffuse to the correct field lines at the sun or, more simply, particle injection occurs after electromagnetic emission.

Fan, Pick, Pyle, Simpson and Smith (1968) have reported solar proton (0.6 13.0 Mev) events during 1966 and have found travel times of typically $10^{4}$ $\left( \pm 2 \times 10^{3}\right)$ seconds. We illustrate in Figure 3 how the time of flight of a $13.0 \mathrm{Mev}$ proton in the simple spiral field would vary with the solar wind velocity $v_{p}$ from Equation (31). The flattening of the curve at high $V_{p}$ illustrates the straightening of spiral field lines. Once again, we emphasize that these results are a lower bound on the problem. The remarks of the above paragraph also apply here. 


\section{B. Azimuthal Motion}

A calculation of the azimuthal motion is most easily done in a reference frame which is rotating with the sun. If $\mathrm{V}_{\mathrm{r}}^{\prime}$ and $\mathrm{V}_{\phi}^{\prime}$ denote the velocity components of the guiding center in this frame, then

$$
\begin{aligned}
v_{r}^{\prime}=v_{r} & =\frac{V_{11} a}{\left(r^{2}+a^{2}\right)^{1 / 2}}+\frac{v_{p} r^{2}}{\left(r^{2}+a^{2}\right)}=\frac{1}{\left(r^{2}+a^{2}\right)^{1 / 2}}\left[V_{11} a+\frac{v_{p} r^{2}}{\left(r^{2}+a^{2}\right)^{1 / 2}}\right] \\
V_{\phi}^{\prime} & =v_{\phi}-\Omega r \sin \theta=-\frac{V_{11} r}{\left(r^{2}+a^{2}\right)^{1 / 2}}+\frac{V_{p} a r}{r^{2}+a^{2}}-\Omega r \sin \theta \\
& =-\frac{r}{a\left(r^{2}+a^{2}\right)^{1 / 2}}\left[V_{11} a+\frac{V_{p} r^{2}}{\left(r^{2}+a^{2}\right)^{1 / 2}}\right]
\end{aligned}
$$

Clearly,

$$
\frac{V_{\phi}^{\prime}}{V_{r}^{\prime}}=-r / a=B_{\phi} / B_{r}
$$

which shows that the guiding center trajectory moves out a spiral magnetic field line when viewed in the rotating (solar) frame. This occurs because there is no electric field in the rotating frame and, hence, no electric drift. An inertial observer would describe the motion as analogous to that of a bead sliding outward along a spiral wire, which is rotating with the angular velocity $\Omega$, or would say that the particle's $\phi$ is the $\phi$ of the spiral field line minus the $\phi$ through which the sun has rotated during the particle's flight. This result may be expressed analytically as

$$
\phi-\phi_{0}=\frac{\Omega \sin \theta}{\mathrm{v}_{\mathrm{p}}}\left[\mathrm{r}_{0}-\mathrm{r}\right]+\Omega \mathrm{t}(\mathrm{r})
$$


with $t(r)$ being given by Equation (31) and $\phi_{0}$ being $\phi$ at $r_{0}$; it is shown in Figure 4 . We note that a solar wind particle, which is injected radially at $r_{0}$, with $\mathrm{V}_{\mathrm{T}}=\mathrm{V}_{\mathrm{p}}$, has $\phi=\phi_{0}$.

The last term in Equation (36) is important if the particle energy is small or if the field is tightly coiled, that is, if $V_{p}$ is small or $r$ is large. For most cases, however, we are justified in ignoring the last term as well as $r_{0}$. Then, if a particle is received at earth, we can say, within our approximate model, that the flare causing the particle injection occurred at

$$
\phi_{0}=\Omega \mathrm{r} N_{\mathrm{p}} \quad \text { West }
$$

This is an equation that has been used in the past but now it has somewhat more justification. Lin and Anderson (1967) have shown that the average heliographic longitude for simple (using their terminology) electron events is $58^{\circ}$ West. This, from Equation (37), would correspond to an average solar wind velocity of about $425 \mathrm{~km}-\mathrm{sec}^{-1}$, quite a reasonable value for solar minimum.

\section{Particle Anisotropy}

We have shown in the previous section that particles move out the magnetic field lines upon which they were injected. As a last point, we show how the particles are rapidly channelled down the magnetic field lines. The average pitch angle $\bar{\psi}$ seen by a stationary observer is given by

$$
\sin ^{2} \bar{\psi}=\left(\mathrm{V}_{\mathrm{c}}^{2}+\mathrm{V}_{\mathrm{d}}^{2}\right) / \mathrm{V}_{\mathrm{T}}^{2}
$$

Using Equations (21) and (26), we may write 


$$
\bar{\psi}=\sin ^{-1}\left\{\left[\frac{\mathrm{r}_{0}^{2}}{\mathrm{r}^{2}}\left(\frac{\mathrm{r}^{2}+\mathrm{a}^{2}}{\mathrm{r}_{0}^{2}+\mathrm{a}^{2}}\right)^{1 / 2} \sin ^{2} \psi_{0}+\frac{\mathrm{r}^{2} \mathrm{~V}_{\mathrm{p}}^{2} / \mathrm{V}_{\mathrm{T}}^{2}}{\mathrm{r}^{2}+\mathrm{a}^{2}}\right]^{1 / 2}\right\}
$$

We see that at $r=r_{0}, \bar{\psi} \approx \psi_{0}$ and at $r>>a, \bar{\psi} \approx \sin ^{-1}\left(V_{p} / V_{T}\right)$. The first term is energy independent and measures the effect of $B(r)$ on $V_{c}(r)$. The complete behavior of $\bar{\psi}$ as a function of $r$ for $V_{p}=500 \mathrm{~km}-\mathrm{sec}^{-1}$ is shown in Figure 5 , where the asymptote of $\sin ^{-1}\left(V_{p} / V_{T}\right)$ is clearly small. Hence at the earth, any unscattered particles should have a highly anisotropic distribution; the anisotropy axis should lie near the field direction. These conclusions are in substantial agreement with the experimental results of Fan et al. (1968) and McCracken, Rao and Bukata (1967).

\section{SUMMARY}

We have investigated the dynamics of an energetic charged particle in a spiral model of the interplanetary magnetic field. After showing that the electric drift velocity is the only large drift velocity, we were able to arrive at approximate analytic solutions for the adiabatic motion of the guiding center of the particle.

These solutions show that the guiding center of the particle moves like a bead sliding outward along a spiral wire which is rotating with the angular velocity of the sun. The spiral wire represents the field line on which the particle starts. A reasonably simple expression is presented for the time of flight, which is inversely proportional to the total particle velocity but which has a more complex dependence upon the distance from the sun, the solar wind 
velocity and the initial pitch angle. Our calculations indicate that the particles have a highly anisotropic distribution upon arrival at the vicinity of the earth.

The above findings are shown to be in substantial agreement with experimental data on unscattered solar cosmic rays. The expressions presented generalize and lend physical interpretation to the computer results of Winge and Coleman (1968). 


\section{APPENDIX: EVALUATION OF $\mu$}

The magnetic moment $\mu$ is a measure of the flux enclosed when the particle circles the guiding center. As an adiabatic invariant of the motion, it is a powerful tool in solving for the motion along the field lines. The classical description is

$$
\mu=\mathrm{mV}_{\mathrm{c}}^{2} / 2 \mathrm{~B}=\mathrm{m} \mathrm{V}_{\mathrm{T}}^{2} \sin ^{2} \psi / 2 \mathrm{~B}
$$

The last step is only true when $\mathrm{V}_{\mathrm{d}}<\mathrm{V}_{\mathrm{T}} \sin \psi$. At other times care must be exercised. We define $\mu$ in terms of the instantaneous vector velocity perpendicular to the field line $V_{I_{I}}$

$$
\mu=\mathrm{m}\left(\mathrm{V}_{\mathrm{I}_{1}}-\mathrm{V}_{\mathrm{d}}\right)^{2} / 2 \mathrm{~B}
$$

The case of zero magnetic moment arises when the particle is injected with just the drift velocity $\left(V_{I_{1}}=V_{d}\right)$. This is of particular interest; the particle does not gyrate at all, is unaffected by a gradient B drift and continues to move across the field at $v_{d}$. This illustrates how a solar wind particle is permitted to move across the interplanetary magnetic field.

There is some ambiguity in Equation (A-2) because $V_{I_{\perp}}$ is usually defined in terms of only an angle $\psi_{0}$ and an energy. Thus the direction of $v_{I_{\perp}}$ is unknown; we arbitrarily choose it to lie in the positive $\phi$ direction in the $(r, \phi)$ plane.

The magnitude of $V_{I_{I}}$ is $V_{T} \sin \psi$, which will dominate $V_{d}$ in this problem for all pitch angles except when $\psi \sim 0\left(V_{d} / V_{T}\right)$. It must be emphasized, however, 
that this is a weak restriction. Thus for almost all cases Equation (A-1) is an accurate expression for the magnetic moment. 


\section{ACKNOWLEDGMENTS}

We thank Dr. E. C. Roelof for his encouragement. Each author holds a National Academy of Sciences-National Research Council Resident Research Associateship supported by NASA. 


\section{REFERENCES}

Alfvên, H. and C. G. Fälthammar, Cosmical Electrodynamics (2nd edition), Clarendon Press, Oxford, 1963.

Fan, C. Y., M. Pick, R. Pyle, J. A. Simpson and D. R. Smith, Protons Associated with Centers of Solar Activity and Their Propagation in Interplanetary Magnetic Field Regions Corotating with the Sun, JGR, 73, 1555, 1968.

Lin, R. P. and K. A. Anderson, Electrons $>40 \mathrm{Kev}$ and Protons $>500 \mathrm{Kev}$ of Solar Origin, Solar Physics, 1, 446, 1967.

McCracken, K. G., U. R. Rao, and R. P. Bukata, Cosmic-Ray Propagation Processes, 1, A Study of the Cosmic-Ray Flare Effect, JGR, 72, 4293, 1967.

Northrop, T. G., The Adiabatic Motion of Charged Particles, Interscience Pub1ishers, New York, 1963.

Parker, E. N., Dynamics of the Interplanetary Gas and Magnetic Fields, Ap.J., $\underline{128}, 664,1958$.

Winge, C. R., Jr. and P. J. Coleman, Jr., The Motion of Charged Particles in a Spiral Field, JGR, 73, 165, 1968. 


\section{FIGURE HEADINGS}

Figure 1. Spiral field configuration, showing parameters and coordinate system.

Figure 2. Time of flight versus $r$ for protons injected at $r_{0}$.

Figure 3. Time of flight for a proton injected at $r_{0}$ to reach 1 A.U. versus the solar wind velocity $\mathrm{V}_{\mathrm{p}}$.

Figure 4. Actual proton trajectory. Dots show the position of the particle in intervals of $10^{4}$ seconds.

Figure 5. Average pitch angle versus $r$ for protons injected at $\mathbf{r}_{0}$. Note that $\bar{\psi}$ decreases rapidly with increasing radius. 


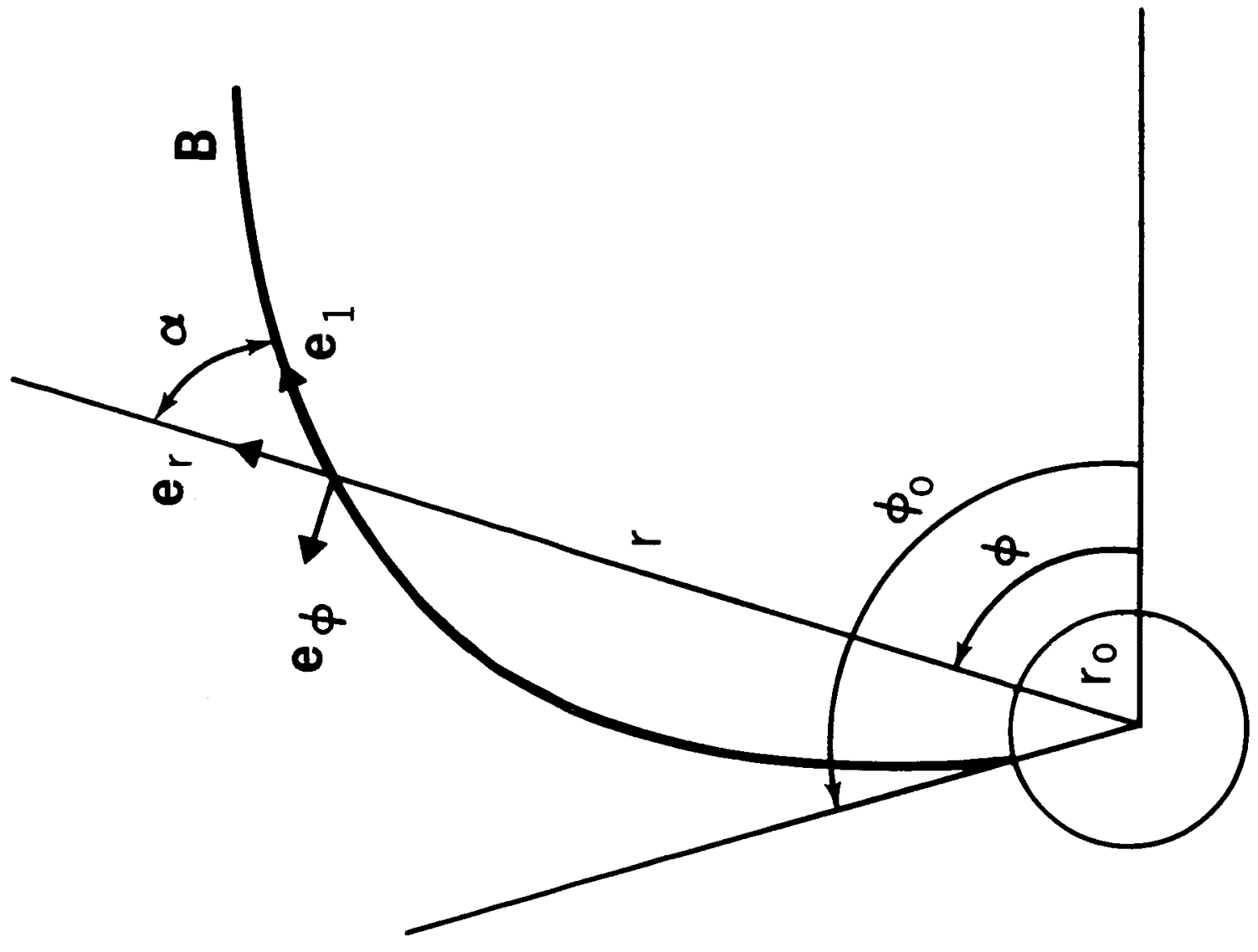




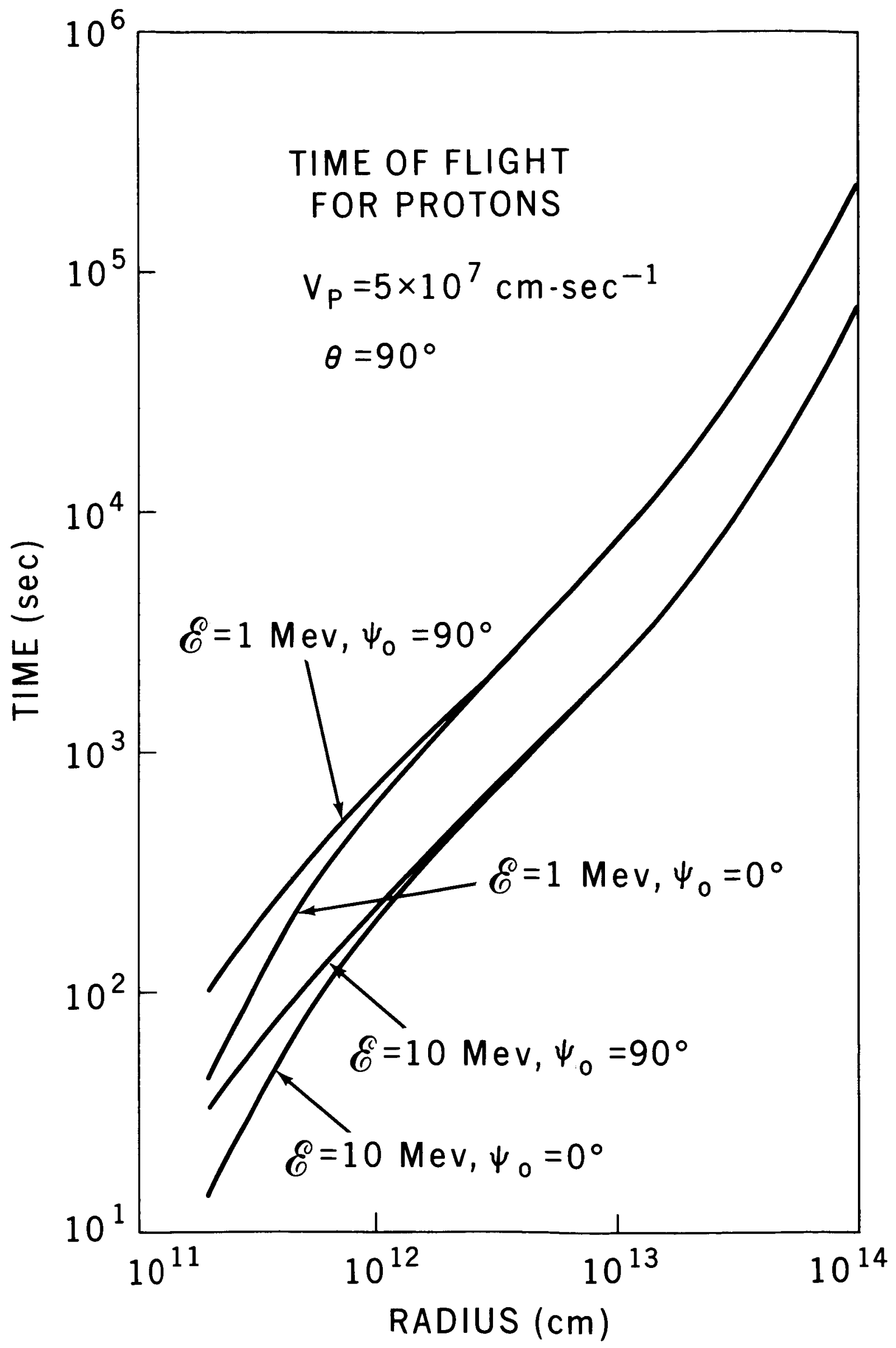




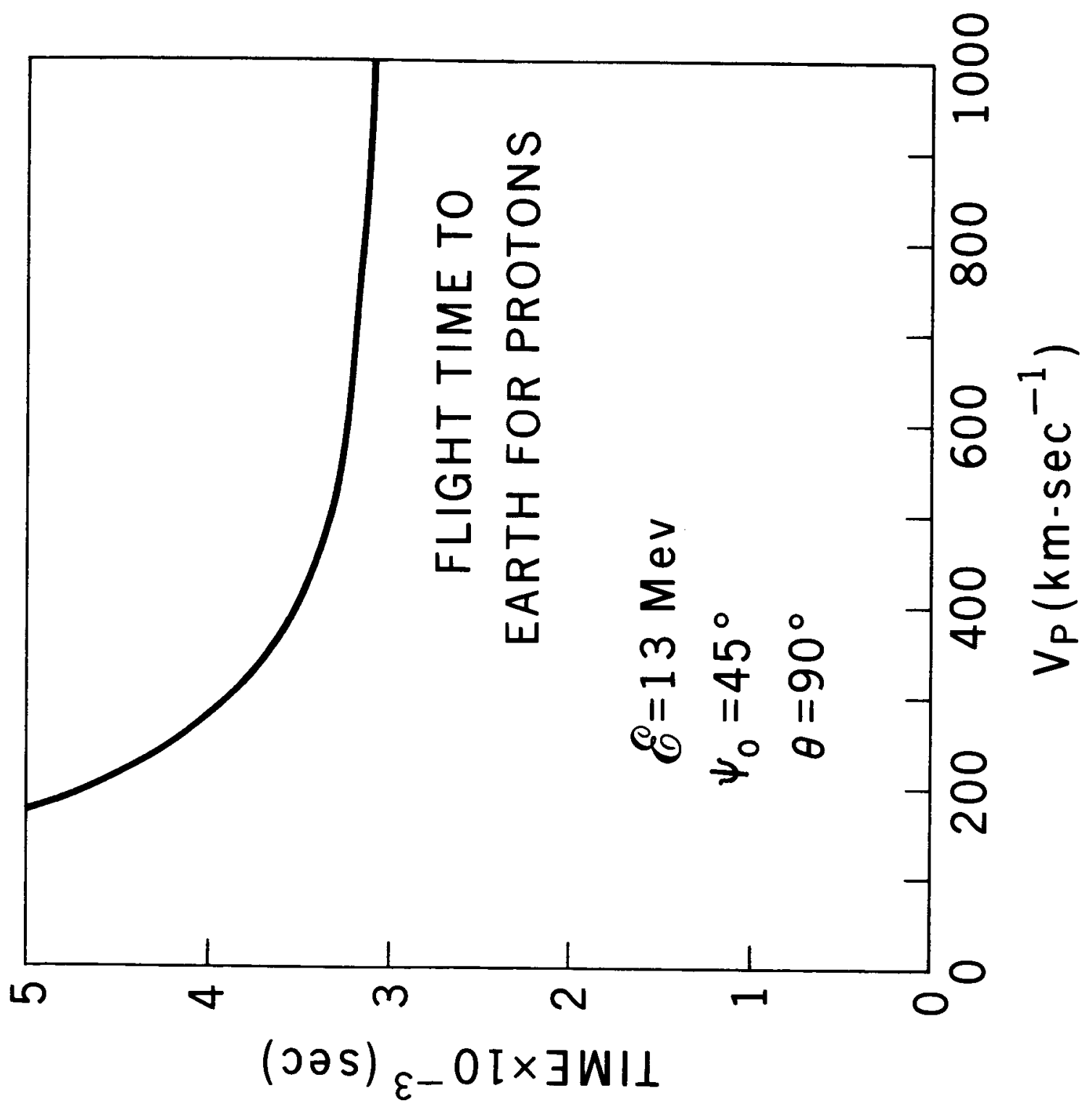




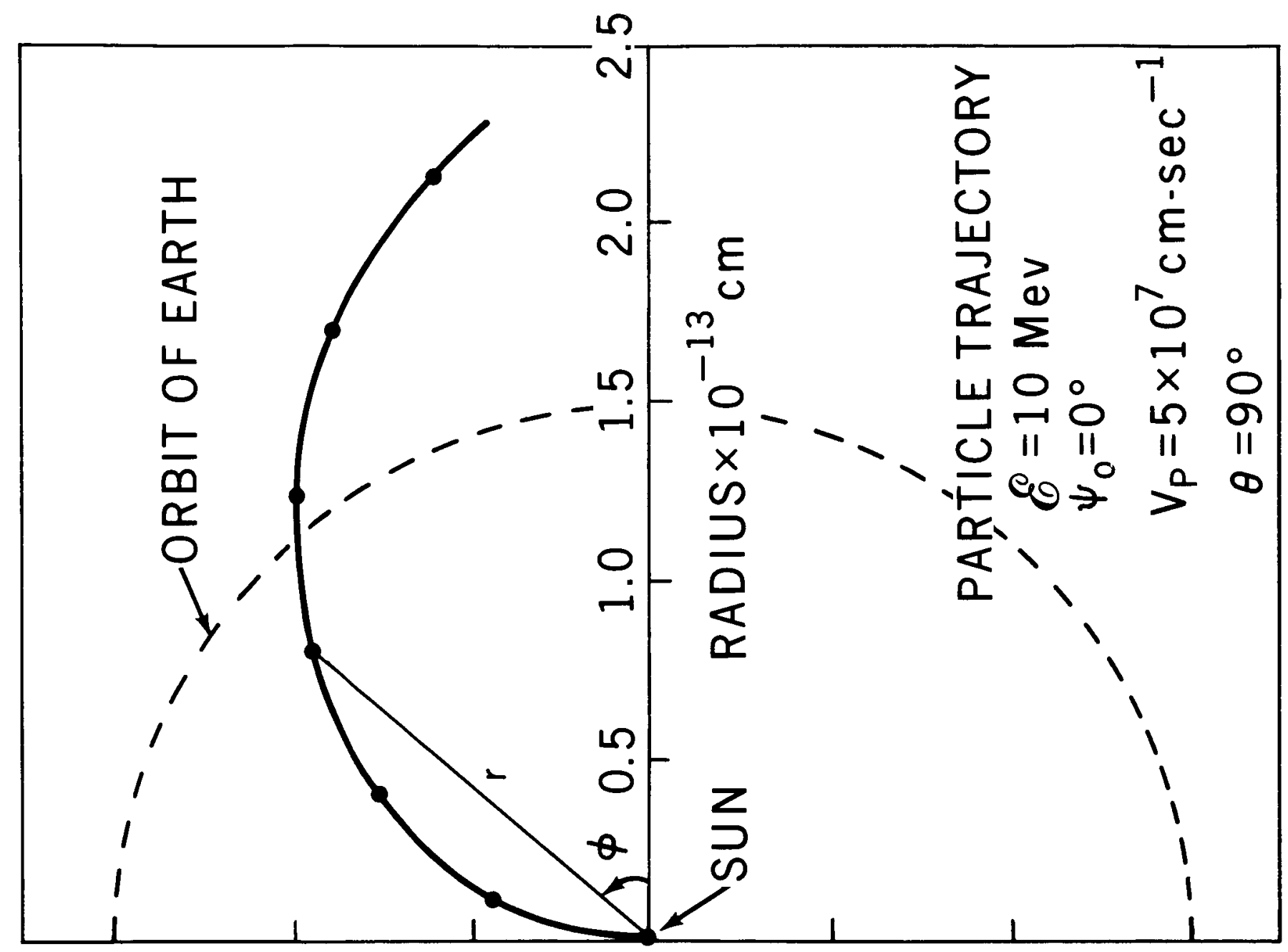




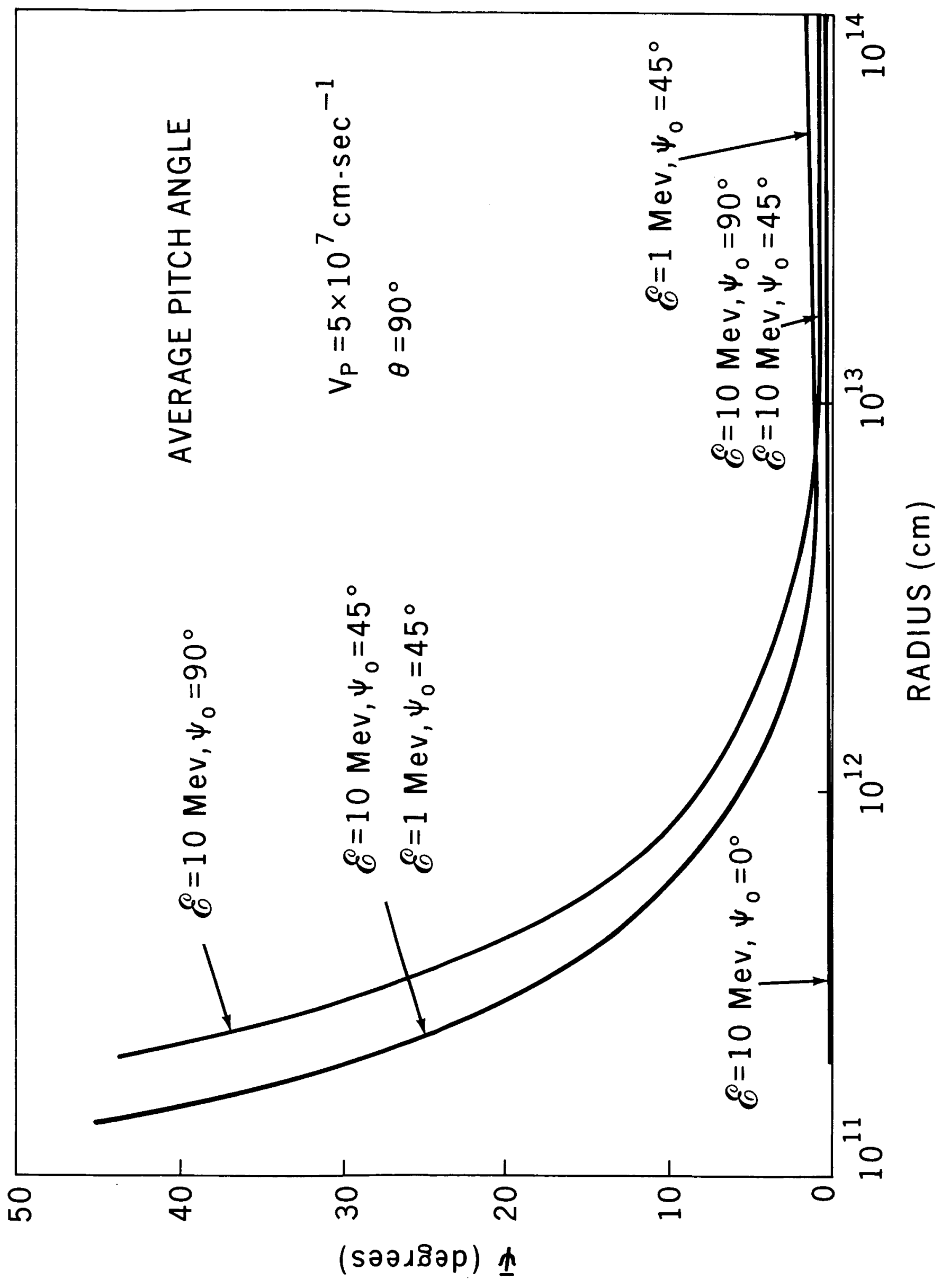

